the study conduct timeline. METHODS/STUDY POPULATION: This study compares twenty Phase 1 COG Pilot and Phase 1 Consortium trials that employed the Rolling 6 design with hypothetical results under the assumption that a $3+3$ design had been executed. The number of evaluable patients required to complete the study, number of DLTs, number of inevaluable patients, overall study duration, time suspended to enrollment (i.e., waiting for DLT evaluation), and DLT risk are compared between study designs using Wilcoxon's signed rank test. RESULTS/ANTICIPATED RESULTS: The Rolling 6 study design required less time to complete the studies compared with $3+3$ design (median 273 vs. 297 days, $P=0.01$ ). In general, the Rolling 6 study design required more patients, had more inevaluable patients, and there were more dose limiting toxicity (DLT) events. However, there was no significant difference in DLT risk (median 0.15 vs. $0.17, \mathrm{P}=0.72$ ). DISCUSSION/SIGNIFICANCE OF IMPACT: The Rolling 6 study design effectively shortens the study conduct timeline compared with the traditional $3+3$ design for Phase 1 COG Pilot and Phase 1 Consortium trials without increasing the risk of toxicity.

3377

A cross-sectional analysis of opioid prescribing patterns among gynecologic oncologists using Medicare fee-for service provider utilization \& payment data

David Samuel ${ }^{1}$, Devin Miller, Sara Isani, Dennis Kuo and Gregory Gressel

${ }^{1}$ Albert Einstein College of Medicine

OBJECTIVES/SPECIFIC AIMS: Opioids are the first-line treatment for moderate to severe cancer-related pain. Increased awareness of opioid prescription misuse and adverse outcomes has prompted statements on their use from multiple national medical groups. In this study we characterize national-level opioid prescription patterns among gynecologic oncologists treating Medicare beneficiaries. METHODS/STUDY POPULATION: The Centers for Medicare and Medicaid Services (CMS) database was used to access Medicare Part D beneficiary data (2016). All available opioid claims prescribed by gynecologic oncologists were identified. Medication type, prescription length and other prescribing factors were recorded. Physician demographics were obtained from departmental websites and accrediting bodies. Physicians with $<10$ opioid claims are not included in the CMS database. Bivariate statistical analysis including chi-squared, Fisher's exact test and Wilcoxon rank-sum test were performed to compare variables with threshold for significance set at $\mathrm{p}<0.05$. Linear regression modeling was also performed to examine association of gender with number of opioids prescribed. RESULTS/ANTICIPATED RESULTS: A total of 494 board-certified gynecologic oncologists were included in this analysis. In 2016, gynecologic oncologists wrote 23,584 opioid prescriptions for 267,824 days of treatment (average of 9.24 prescribed days per claim). The most commonly prescribed opioid was oxycodone/ acetaminophen (41\%). Male physicians had significantly more opioid prescription claims than females $(\mathrm{p}<0.01)$ including after adjusting for differences in years of experience. The majority of physicians had 11-50 opioid prescription claims (68\%). A minority were high prescribing physicians with $>100$ opioid claims (11\%). Of these, the overwhelming majority were male $(82 \%)$ and late career $(46 \%$, $>15$ years since board certification). Physicians in the South had the greatest number of opioid prescription claims and significantly more than physicians in the Northeast, who had the fewest $(\mathrm{p}<0.01)$. Mean number of opioid claims increased with increasing years of experience $(p<0.05)$. DISCUSSION/SIGNIFICANCE OF IMPACT: Among gynecologic oncologists, there were genderbased, regional and experience-related variations in opioid prescribing in the Medicare population in 2016. Further longitudinal studies are required to elucidate secular trends in opioid prescription practice.

\section{A Randomized Controlled Trial Comparing the Nonabsorbable Antibiotic Rifaximin vs. Dietary Intervention Low in Fermentable Sugars (FODMAP) in Irritable Bowel Syndrome \\ Allen Lee ${ }^{1}$, Krishna Rao ${ }^{1}$, Emily Haller ${ }^{1}$, Lauren Van Dam ${ }^{1}$, Jason Baker ${ }^{1}$, Shanti Eswaran ${ }^{1}$, William Chey ${ }^{1}$, Vincent Young ${ }^{1}$, Chung Owyang ${ }^{1}$ and William Hasler ${ }^{1}$ \\ ${ }^{1}$ University of Michigan School of Medicine}

OBJECTIVES/SPECIFIC AIMS: Objectives and goals of this study are to (i) determine whether IBS-D patients randomized to either rifaximin or low FODMAP diet show improvement in IBS-related symptoms; and (2) identify using longitudinal analyses how SIBO status and fecal microbiota features associate with response to either rifaximin or low FODMAP dietary intervention. METHODS/ STUDY POPULATION: 42 patients $\geq 18$ years of age who meet Rome IV criteria for IBS-D will be randomized to receive either rifaximin or low FODMAP diet intervention. The primary outcome will be the proportion of responders to intervention which is defined as $\geq 30 \%$ reduction in mean daily abdominal pain or bloating by visual analog scale compared with baseline. Exclusion criteria will include: (a) history of microscopic colitis, inflammatory bowel disease, celiac disease, or other organic disease that could explain symptoms, (b) prior gastrointestinal surgery, other than appendectomy or cholecystectomy $>6$ months prior to study initiation, (c) prior use of rifaximin or formal dietary interventions for IBS-D, (d) use of antibiotics within the past 3 months, or (e) use of probiotics within 1 month of study entry. Glucose hydrogen breath tests will be performed at the beginning and end of the trial to evaluate for SIBO. Fecal samples will be collected at 0,2 , and 6 weeks to determine changes in fecal microbial composition and structure. RESULTS/ ANTICIPATED RESULTS: This study seeks to examine whether longitudinal analyses of small intestinal and colonic microbiota can subtype IBS-D subjects into clinically relevant phenotypes. A total of 18 subjects have been enrolled into the study. Clinical variables, hydrogen breath test results, and fecal microbiota data are being collected for ongoing analysis. DISCUSSION/ SIGNIFICANCE OF IMPACT: Results from this study may help move treatment of IBS from a purely symptom based approach to a more individualized approach by stratifying IBS-D patients into distinct clinical phenotypes which are amenable to targeted therapeutic approaches.

\section{5}

A1BG and ITIH4 proteins are upregulated on HDL of youth with type 1 diabetes and correlate with glycemic control Evgenia Gourgari ${ }^{1}$, Scott Gordon, Junfeng Ma, Martin Playford, Nehal Mehta, Radoslav Goldman and Alan Remaley

${ }^{1}$ Georgetown - Howard Universities

OBJECTIVES/SPECIFIC AIMS: Our objective was to compare the proteomics of HDL between youth with T1DM and healthy controls 
(HC). METHODS/STUDY POPULATION: We did chromatography-based HDL purification and SWATH-MS-based proteomic quantitation. Proteomic alterations of HDL fractions and their association with glycemic control was examined. Study population: 26 patients with T1DM and 13 HC. RESULTS/ANTICIPATED RESULTS: We quantified 78 proteins in isolated HDL, using mass spectrometry and label-free SWATH quantification. Youth with T1DM had significantly higher protein levels of A1BG $(P=0.008)$, A2AP $(\mathrm{P}=0.0448)$, APOA4 $(\mathrm{P}=0.0366)$, CFAH $(\mathrm{P}=0.0476)$, FHR2 $(\mathrm{P}=0.0005), \mathrm{ITIH} 4(\mathrm{P}=0.01), \mathrm{PGRP} 2(\mathrm{P}=0.0167)$ and lower levels of ALBU $(\mathrm{P}=0.0164)$ and $\mathrm{CO} 3(\mathrm{P}=0.019)$ compared to HC. A1BG $(\mathrm{r}=0.541, \mathrm{P}<0.001)$ and ITIH4 $(\mathrm{r}=0.357, \mathrm{P}=0.026)$ were significantly positively correlated with HbA1c. DISCUSSION/SIGNIFICANCE OF IMPACT: Youth with T1DM have proteomic alterations of their HDL compared to HC, despite similar concentration of HDL cholesterol, that might affect the cardioprotective mechanisms of HDL. Future efforts should focus on investigating the role of these HDL associated proteins in regard to HDL function and their role in CVD risk in patients with T1DM.

Academic influence in gynecologic oncology is associated with industry funding: an analysis of the Open Payments database

David Samuel, Shelby Adler, Nicole Vilardo and Gregory Gressel Albert Einstein College of Medicine

OBJECTIVES/SPECIFIC AIMS: Industry payments to physicians can present a conflict of interest. The Physician Payments Sunshine Act mandates the disclosure of these financial relationships to increase transparency. Recent studies in other surgical specialties have shown that research productivity is associated with greater industry funding. In this study, we characterize the relationship between academic influence and industry funding among academic gynecologic oncologists. METHODS/STUDY POPULATION: Departmental websites were used to identify academic gynecologist oncologists and their demographic information. The Hirsch index (h-index) relates an author's number of publications to number of times referenced by other publications, a validated measure of an author's academic influence. This was obtained from the Scopus database. The Center for Medicaid and Medicare Services Open Payments online database was searched for all industry payments in 2017. The NIH Reporter online database was searched for active grants. Goodness of fit testing showed that all variables followed nonparametric distributions. Medians were compared using MannWhitney $U$ tests and Kruskal-Wallis analysis of variance with post-hoc Dunn's test. RESULTS/ANTICIPATED RESULTS: Four hundred and sixty-six academic gynecologic oncologists were included in the analysis. In $2017,89.7 \%$ of this group received industry funding totaling $\$ 41.4$ million. Median industry funding was $\$ 453$ [IQR \$67-19684] and median h-index was 14 [IQR 8-26]. Only $8.1 \%$ of gynecologic oncologists were NIH grant recipients and they received significantly higher industry payments ( $\$ 357$ vs. 11,168 , $\mathrm{P}<0.01$ ). Gender and academic rank were not associated with industry funding. Gynecologic oncologists in the highest decile of industry funding received a median payment of $\$ 447,651[\mathrm{~N}=46$, IQR $\$ 285,770$ - 896,310] totaling $\$ 36.5$ million. The median h-index for this top-earning decile was 23 [N=46, IQR 16.5-30.3]. When stratified by payment amount, median $\mathrm{h}$ index increased but only reached statistical significance in the highest cohort receiving $>\$ 100,000(\mathrm{~N}=63, \mathrm{P}<0.05)$. DISCUSSION/SIGNIFICANCE OF
IMPACT: The majority of academic gynecologic oncologists receive industry funding although there are large variations in payments. Those receiving the largest payments are more likely to hold NIH grants and have greater academic influence.

3577

Adiposity and Fibroblast Growth Factor 23 in nondiabetic patients with moderate-to-severe Chronic Kidney Disease

Elvis Akwo ${ }^{1}$, Cassiane Robinson-Cohen ${ }^{1}$, Aseel Alsouqi ${ }^{1}$, Edward Siew ${ }^{1}$, Alp Ikilzer ${ }^{1}$ and Adriana Hung ${ }^{1}$

${ }^{1}$ Vanderbilt University Medical Center

OBJECTIVES/SPECIFIC AIMS: The main aim of this study was to investigate the relationship between measures of adiposity and FGF-23 in a sample of patients with CKD stages 3-4. METHODS/STUDY POPULATION: This study was a clinic-based cross-sectional investigation of $71 \mathrm{CKD}$ patients who underwent body composition and anthropometric assessments as part of the relationship of insulin sensitivity in kidney disease and vascular health (RISKD) study. Dual energy x-ray absorptiometry (DEXA) scans were used to measure total fat mass and body mass index (BMI) was computed using baseline weight and height measurements. Biomarkers included serum FGF-23 (C-terminal), serum leptin, high sensitivity C-reactive protein (hsCRP), serum triglycerides, high density lipoprotein (HDL) cholesterol and total cholesterol. Creatinine-based estimated glomerular filtration rate (eGFR) was computed using the CKD-EPI equation. Multiple linear regression with robust standard errors was used to investigate the relationship between FGF2-3 and measures of adiposity (BMI, total fat mass and serum leptin). Log-transformation was performed for variables (FGF-23, hsCRP and serum lipids) with considerable skewness. RESULTS/ANTICIPATED RESULTS: The median age of the study participants was 68 (IQR: 60, 73) years; $26 \%$ were female and $23 \%$ were African-American. Median eGFR was $46.9 \mathrm{ml} / \mathrm{min} / 1.73 \mathrm{~m} 2$ (IQR: $41.9,52.8$ ), median BMI was $31 \mathrm{~kg} / \mathrm{m} 2$ (IQR: 27, 35). Log FGF-23 had a significant positive correlation with BMI $(r=0.27, p=0.02)$, total fat mass $(r=0.30$, $\mathrm{p}=0.01)$ and serum leptin $(\mathrm{r}=0.43, \mathrm{p}<0.0001)$. After full adjustment for age, sex, race, eGFR, log hsCRP, log HDL and log triglycerides, a $50 \%$ increase in FGF-23 was associated with a $1 \mathrm{~kg} / \mathrm{m} 2$ [95\% CI: 0.1, 1.9; $\mathrm{p}=0.03$ ] increase in BMI, a $2.5 \mathrm{~kg}$ [95\% CI: 0.2, 4.8; $\mathrm{p}=0.03]$ increase in total fat mass and a $6.7 \mathrm{ng} / \mathrm{mL}$ [95\% CI: 1.0, 12.4; $\mathrm{p}=0.02]$ increase in serum leptin. DISCUSSION/SIGNIFICANCE OF IMPACT: In this sample of patients with moderate-to-severe CKD, we found a significant independent association between higher FGF-23 levels and higher adiposity (BMI, total fat mass and the proatherogenic adipocytokine, leptin). The underlying causes and the implications of these associations - particularly in bone and vascular health - need to be further investigated.

3469

Age and racial variation in the relation between blood lead level and asthma in children: Data from National Health and Nutrition Examination Survey 1999-2016

Magda Shaheen ${ }^{1}$ and Deyu Pan

${ }^{1}$ David Geffen School of Medicine at UCLA

OBJECTIVES/SPECIFIC AIMS: Lead $(\mathrm{Pb})$ exposure can seriously affect nervous system and kidney. Young children are vulnerable to $\mathrm{Pb}$ exposure. However, the role of low-level $\mathrm{Pb}$ exposure in asthma 